\title{
Automation Accuracy Is Good, but High Controllability May Be Better
}

\author{
Quentin Roy \\ School of Computer Science \\ University of Waterloo \\ quentin@quentinroy.fr
}

\author{
Futian Zhang \\ School of Computer Science \\ University of Waterloo \\ chzft333@gmail.com
}

\author{
Daniel Vogel \\ School of Computer Science \\ University of Waterloo \\ dvogel@uwaterloo.ca
}

\begin{abstract}
When automating tasks using some form of artificial intelligence, some inaccuracy in the result is virtually unavoidable. In many cases, the user must decide whether to try the automated method again, or fix it themselves using the available user interface. We argue this decision is influenced by both perceived automation accuracy and degree of task "controllability" (how easily and to what extent an automated result can be manually modified). This relationship between accuracy and controllability is investigated in a 750-participant crowdsourced experiment using a controlled, gamified task. With high controllability, self-reported satisfaction remained constant even under very low accuracy conditions, and overall, a strong preference was observed for using manual control rather than automation, despite much slower performance and regardless of very poor controllability.
\end{abstract}

\section{KEYWORDS}

accuracy; controllability; automation; controlled experiment

\section{ACM Reference Format:}

Quentin Roy, Futian Zhang, and Daniel Vogel. 2019. Automation Accuracy Is Good, but High Controllability May Be Better. In CHI Conference on Human Factors in Computing Systems Proceedings (CHI 2019), May 4-9, 2019, Glasgow, Scotland UK. ACM, New York, NY, USA, 8 pages. https://doi.org/10.1145/3290605.3300750

Permission to make digital or hard copies of all or part of this work for personal or classroom use is granted without fee provided that copies are not made or distributed for profit or commercial advantage and that copies bear this notice and the full citation on the first page. Copyrights for components of this work owned by others than the author(s) must be honored. Abstracting with credit is permitted. To copy otherwise, or republish, to post on servers or to redistribute to lists, requires prior specific permission and/or a fee. Request permissions from permissions@acm.org. CHI 2019, May 4-9, 2019, Glasgow, Scotland UK

(c) 2019 Copyright held by the owner/author(s). Publication rights licensed to ACM.

ACM ISBN $978-1-4503-5970-2 / 19 / 05 \ldots \$ 15.00$

https://doi.org/10.1145/3290605.3300750

\section{INTRODUCTION}

Machine learning, artificial intelligence, computer vision and other advanced form of automation ${ }^{1}$ are more and more integrated in every day tasks with the promise to reduce workload and improve productivity. However, accurate automation is difficult to do perfectly and some amount of inaccuracy is virtually unavoidable. Even if rare, it can cause great frustration if users do not have sufficient leverage to rectify and fix the result. For example, using a mobile intelligent assistant for some tasks can feel poor, even when speech recognition quality is reasonably high. A possible reason is that even a single inaccurate word requires irritatingly tedious manual edits of recognized text.

When faced with inaccurate results, users have essentially three rectification options: (1) try the automation again hoping for a better result ("auto" approach); (2) try to fix the result themselves using a user interface ("manual"); or (3) a combination of both ("mixed"). We argue this decision is influenced by how much control any corresponding user interface provides to manipulate the task result, including its usability $[6,19]$. We call this the "controllability" of the automated task, and we study its relationship with accuracy.

While considerable resources are focused on improving automation, its relationship to controllability is not often considered in the context of automated tasks [15]. In fact, the informal motto of many automation communities is that the user cannot help, and user-in-the-loop approaches are generally considered failures [22]. Without diminishing the importance of improving accuracy, we believe an uncompromising research direction seeking only higher accuracy is hurtful to the design of usable automated systems, and we argue that user acceptance of automation accuracy [9] is strongly related to controllability.

We investigate the relationship between accuracy and controllability in an automated task using objective measures of system usage and subjective user satisfaction. This is achieved with an online crowdsourced experiment that implements a search task represented by a crane simulator (see

\footnotetext{
${ }^{1}$ We use the term "automation" to designate the programming of complex tasks to be automatically executed by a machine.
} 
Fig.1). Automation accuracy is controlled by varying how accurately the crane is able to autonomously stack boxes, and task controllability is controlled by varying the amount of effort required by the user to manually fix inaccuracies. Results show that even with low accuracy, self-reported satisfaction remains constant for high controllability conditions, and vice versa. It increases with both accuracy and controllability on the rest of the range. Also, a strong preference for manual control was observed, even under very poor controllability and high accuracy conditions. Our contributions includes a formalization of the concept of controllability in an automated task; and insights on the relationship of automation accuracy and controllability on usage and satisfaction.

\section{BACKGROUND AND DEFINITIONS}

Before describing our experiment, we summarize the autonomy versus control debate, provide a clear definition for controllability, and discuss work about acceptable accuracy.

\section{Machine Autonomy versus Control}

The main promise of better automation is the reduction of humanity's workload. When integrated in a user's task, and according to the dominant vision amongst the HCI community, automation should be part of, and designed as a $t o o l^{2}$ to augment human intellect [17]. On the other hand, automation communities aim at creating partners $^{2}$ that entirely carry out tasks on a user's behalf [12] The user interface is often perceived as necessary, but secondary [15].

The subtlety between the two visions has lead to heated debates between the two communities, an instance of which is Shneiderman and Maes's 1997 discussion [20]. The automation communities strive to make systems more autonomous, which means more independent from users. With more autonomous automation, users do not need to do as much, but generally lose some control in exchange. This has been seen as an unacceptable compromise for HCI researchers, particularly in the 1990s $[10,17,18]$. As an example, Shneiderman argued that users seek a feeling of mastery, accomplishment, and responsibility, not "the sense that some intelligent machine magically did their job for them" [17]. However, this claim has never been formally investigated before our work.

Regardless of disagreement in overall vision, automation accuracy sometimes directly trades off against controllability. For example, the behaviour of machine learning algorithms, such as neural networks, are intrinsically difficult to understand and anticipate, even for experts [1, 15]. In case of inaccuracies, one is often required to tinker with the input of the automation, floundering to get the result they need. These are instances of systems with low controllability. Beyond technological limitations, a trade-off can also be about

\footnotetext{
${ }^{2}$ Two of three interaction paradigms identified by Beaudouin-Lafon [3].
}

resource allocation, or where to focus research efforts: better automation, or better controllability? Our work provides empirical insights that may help resolve this trade-off.

Even though automation researchers ultimately conceded that users need to retain some control [20], relying on the user's help is often perceived as a failure, and user-in-theloop approaches are less frequently published [22]. In parallel, the HCI community increasingly embraces automation.

\section{Controllability of Automated Tasks}

We define an automated task as any task integrating some form of automation, such as face detection, voice recognition, text completion, email filtering, and so on. The results of these automations are not always accurate, and often require some "rectification" to correct the automated result.

Previous works have used the terms "control" or "controllability”. For example Shneiderman argues that users need to be "in control" [17]. This claim tend to be confirmed by the work of $\mathrm{Oh}$ et al., who measured the perceived controllability of a collaborative drawing application, and observed that people generally preferred to take initiative over the automation [14]. However, "controllability" has not been precisely defined, and we are not aware of previous work that systematically investigated the impact of controllability, nor its relationship with accuracy.

We define the controllability of an automated task as how much a user is "in control" of the process. Controllability reflects to what extent they can control the automation or alter its result to reach their goal, and how easily and rapidly can this control be carried out. An example of low controllability can be seen in a photo panorama stitching task. The automation can make large errors, such as distorting the image (e.g. cutting people in half), but manually correcting the mistake is difficult, or even impossible. In this case, the task is a failure, with the only recourse to run the automation again. It can be useful to distinguish three forms of controllability: (a) the input controllability of an automated task, that is the ability to easily tweak the input to the automation to improve the result; (b) the manual controllability, that is the ability to bypass the automation completely to reach the necessary result; and (c) the result controllability, that is the ability to easily adjust and rectify inaccurate automation results. In our experiment, we focus on the latter.

Controllability is closely related to usability since the harder it is to fix inaccuracies, the lower controllability is. Usability is commonly defined as how efficient and effective the system is in the execution of a task $[6,19]$. Though other scales exist [4, 21], the Task Load Index (NASA-TLX) [7] is one of the most popular. It includes six different factors: mental demand, physical demand, temporal demand, (perceived) performances, effort, and frustration level. 


\section{Automated Tasks and Acceptable Accuracy}

The accuracy of an automation represents its ability to produce "correct" results, specifically results as close as possible to the user's need. Kay et al., inspired by the Technology Acceptance Model (TAM) [5], introduced the notion of $a c$ ceptable accuracy [9]. They focus in particular on classifiers, and proposed a survey instrument to measure users' perception of accuracy. Both Kay et al. and Katsuragawa et al. noticed that different recognizers are perceived differently by users $[8,9]$. However, acceptable accuracy was only investigated in the context of automation with which user is passive [9], like notifications, or input recognition $[8,16]$. Automated tasks were not considered, and usability only in the context of input recognition.

\section{EXPERIMENT}

This experiment investigates user perception and rectification strategies of an automated task over a range of automation accuracy levels with different levels of controllability of the automation result.

We designed a minimalist crane simulator as a synthetic automated search task with various degrees of control and accuracy. The automation accuracy is represented by the ability of the crane to automatically locate a target position. We manipulate the controllability by varying the amount of effort needed to rectify the automation results manually. Namely, we vary the number of manual steps that need to be taken to move the crane to the target, more steps implying more effort and lower controllability. The experiment was conducted online using Amazon Mechanical Turk.

\section{Crane Simulator}

Participants were asked to play a crane simulator game in a web browser (Fig. 1). The goal is to use a crane to stack boxes on top of each-other. Each time the crane gets a new box to stack, it moves around by itself to appear as though it is trying to detect the box to stack on to, called the "target segment". As a result, the automation runs at least once every trial. We call the crane automation the "Box Detection System" (BDS). Depending on an ACCuRACy factor, the BDS is not always accurate, and the crane may come to rest away from the target segment. To correct an inaccurate result from the first automation run, the participant has two options: (1) run the BDS again by pressing SPACE, which may or may not result in a better location, or (2) move the crane manually using the LEFT and RIGHT arrow keys. Participants can release the box using the DOWN key, but doing so when the crane is outside the target segment results in a failed trial. Executing a command like move left or re-run the BDS takes time, and no further commands are possible until the crane completes the action.

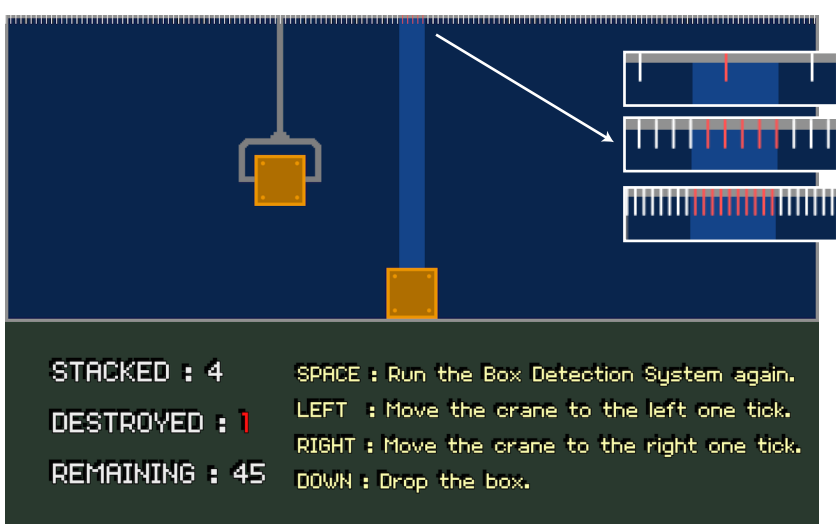

Figure 1: Crane Simulator Task. A light blue area indicates the drop zone for the target segment, and ticks at the top show how far the crane moves with each manual control input. In this figure, magnified boxes illustrate movement step sizes for three control conditions: $32,6.4$, and $3.2 \mathrm{px}$.

\section{Experimental Task}

After a few introduction pages explaining the crane simulator's operation, participants were instructed to play the game for 5 practice trials then 50 measured trials. We logged all events to measure each participant's use of the automation (the BDS) versus manual controls to rectify. After all trials, participants answered a NASA-TLX questionnaire, followed by eight Likert-scale questions about agreement on (1) Perceived Accuracy, (2) Perceived Controllability, (3) Feeling of Accomplishment, (4) Feeling of Control, (5) Feeling of Responsibility, (6) Satisfaction, and (7) Enjoyment. Questions 3 to 5 are derived from Shneiderman's claim that people need control, accomplishment, and responsibility, and that these do not go well with automation [17]. We provide the detailed questionnaire as supplementary material. As an extra precaution, three questions were designed to check participant's attention at two different points in the experiment.

Participants were instructed to minimize the number of failed trials, when the box is released outside the target segment. However, no performance-based bonus reward was given. In practice, participants had a $98 \%$ success rate $(S D=6 \%)$. We attribute this to the gamification of the task, and the mindset of the participants. Mechanical Turk workers tend to optimize the time they spend on each job, and we suspect they assumed that they would not be paid if they failed too many trials. This behaviour is already representative or real-world tasks, and as a result we did not introduce any additional time pressure.

\section{Conditions}

We aim to systematically explore the full space combining accuracy with controllability. This requires pushing each factor to extreme levels. We relate the two conditions by 
the average number of commands that need to be taken to complete a trial using only manual control for rectification (Manual approach), or only by re-running automation (Auto approach). Our manipulations of accuracy and controllability affect both effort and task completion time, and we expect participants to change behaviour accordingly.

Accuracy. The ACCURACY factor affects how far off the automation is, on average, from the target segment. $90 \%$ accuracy means that it effectively positions the crane in the target segment 9 out of 10 times, and that when it misses, it will not be far from the target. However, as the accuracy decreases, more adjustments by the participant is required. In this experiment, the automation result is a normally distributed random variable. Its standard deviation is calculated from ACCURACY $a$ :

$$
\begin{aligned}
\sigma & =\frac{L}{2} \cdot \frac{1}{\sqrt{2} \cdot \operatorname{erf}^{-1}(a)} \\
& =8 \sqrt{2} \cdot \frac{1}{\operatorname{erf}^{-1}(a)}
\end{aligned}
$$

where $\operatorname{erf}^{-1}$ is the inverse error function ${ }^{3}$, and $L$ the length of the target segment: $32 \mathrm{px}$ in our experiment. Consequently, in high ACCURACY conditions, even when out of the target segment, the crane is never far off. We included five ACCURACY values from 0.1 to 0.9 .

Controllability. The controllability is manipulated by varying the STEP of the crane each time a manual command is selected, that is, the distance it travels on each command. The smaller this factor, the less distance the crane moves on each command, and as a result more actions are needed to manually complete a trial. The less accurate the automation is, the further-off its result can be, so the expected number of commands required by a Manual Rectification Approach $\left(\mu_{m}\right)$ depends on both STEP $s$ and ACCURACY $a$ :

$$
\begin{aligned}
\mu_{m} & =E\left(n_{m}\right) \\
& =\frac{m a d}{s} \\
& =\frac{\sqrt{\frac{2}{\pi}} \cdot \sigma}{s} \\
& =\frac{16}{\sqrt{\pi}} \cdot \frac{1}{\operatorname{erf}^{-1}(a) \cdot s}
\end{aligned}
$$

where $n_{m}$ is the number of manual steps, and mad is the mean absolute deviation of the automation from the centre of the target segment.

Note that the STEP cannot be greater that the length of the target segment $L$, or else it may be impossible to manually position the crane inside the target segment. We included 6 STEP values covering a large controllability range: $L=32 \mathrm{px}$, $L / 5=6.4 \mathrm{px},{ }^{L} / 10=3.2 \mathrm{px},{ }^{L} / 20=1.6 \mathrm{px},{ }^{L} / 40=0.8 \mathrm{px}$, which

\footnotetext{
${ }^{3}$ The error function erf $(x)$ describes the probability of a normally distributed random variable with mean 0 and variance $1 / 2$ falling within $[-x, x]$.
}

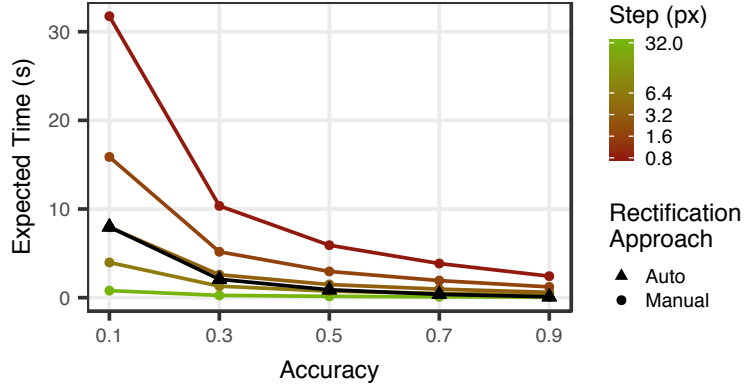

Figure 2: Expected time of the rectification approaches.

ranges from 0.24 steps to be taken on average (ACCURACY $a=0.9$, STEP $s=32$ ) to 130 steps (ACCURACY $a=0.1$, STEP $s=0.8)$. At first, we also included $L / 3=10.7 \mathrm{px}$, but we later removed it to focus on lower controllability values.

An other important aspect is the time taken for each command to be completed. $E\left(t_{a}\right)$ is the expected time to complete a trial using only the automation, and $E\left(t_{m}\right)$ the expected time to complete a trial using only manual controls. We set up the manual movement time per step to $250 \mathrm{~ms}$ to be fast, but with a noticeable delay. We adjusted automation time to be $890 \mathrm{~ms}$ to create the relationship shown in Fig. 2, where:

- $E\left(t_{a} \mid a=0.9\right) \approx E\left(t_{m} \mid a=0.9, s=32\right)$ and,

- $E\left(t_{a} \mid a=0.1\right) \approx E\left(t_{m} \mid a=0.1, s=3.2\right)$.

While this configuration may seem to put the manual control at a significant disadvantage, our pilot experiments revealed people had a strong preference to use manual control, even when automation would be much faster.

\section{Design}

Our experiment was between-subjects with these factors:

- ACCURACy $a=\{0.1,0.3,0.5,0.7,0.9\}$,

- $\operatorname{STEP} s=\{32,6.4,3.2,1.6,0.8\}$.

We aimed for 30 participants $\times 5$ ACCURACY $\times 5$ STEP $=$ 750 participants and 37,500 trials (50 in each condition).

\section{Participants}

We recruited 781 participants using Amazon Mechanical Turk in the United States. To improve reliability, participants were required to have a prior MTurk approval rate of $95 \%$ or higher. 75 participants (not included in the above count) were filtered out just after the instructions since they failed our first attention test. In addition, we did not include the data of 18 participants in the analyses of the questionnaire or demographics, as they responded incorrectly to a second attention question. However, their data remained in our approach usage analyses. Participants were 19 to 75 years old $(N=772$, mean $=35.5, \mathrm{SD}=10.3), 311$ identified as female and 466 as male. Remuneration was $\$ 2.5$ to $\$ 4$, depending on the conditions and the expected completion time. 

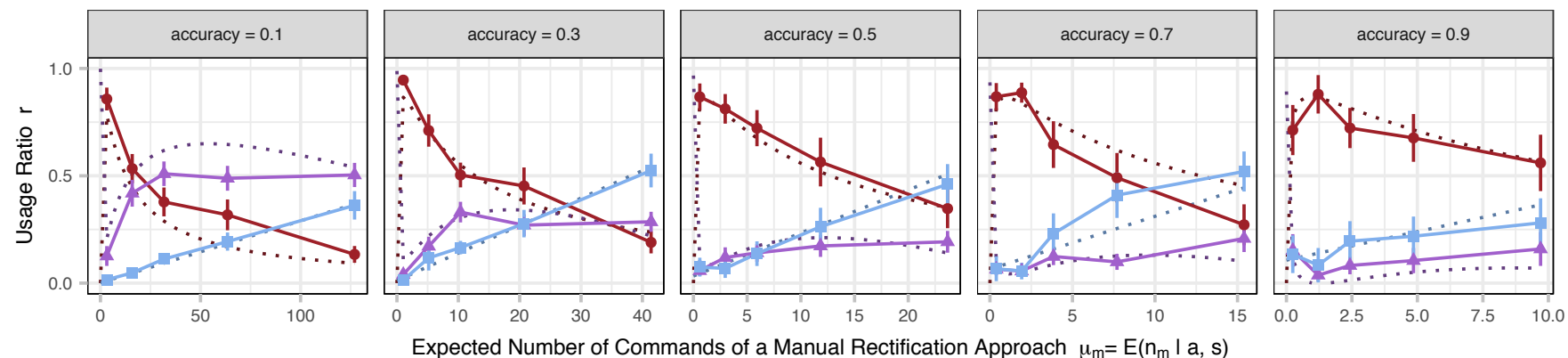

Rectification Approach Manual $\triangle$ Mixed $\square$ Auto

Figure 3: Rectification Approach Uses. Doted lines show the predictions of our models. Note that the scale of the y-axis differ.

\section{RESULTS}

Our results on approach use are shown on Fig. 3. The main dependent variables are the use ratios of the three different rectification approaches: "auto" if the first automation result was rectified using the automation only, "manual" if it was rectified using the manual controls only, and "mixed" otherwise. We are specifically interested in the first two.

Approach ratios are calculated by dividing the number of trials completed using the approach, by the number of all trials that required rectification. This produces three dependent variables per participant, whose sum equals 1: Manual Approach Ratio $\left(r_{m}\right)$, Auto Approach Ratio $\left(r_{a}\right)$ and Mixed Approach Ratio $\left(r_{x}\right)$.

\section{Analysis using Curve-Fitting}

We performed a curve-fitting analysis to provide a structured way to describe and compare the pattern of results. In some ways, this is akin to how one might use a linear regression model instead of significance tests. While our experiment investigated 25 conditions under several metrics, curve fitting enables us to represent our results with two mathematical expressions and nicely complements the data points.

We independently fitted the distribution of manual and auto using both ACCURACY and expected number of manual steps $\mu_{m}=E\left(n_{m} \mid a, s\right)$ as predictors. To do so, first, we coarsely performed Damped Least-Squares regressions [11, 13] on both dependent variables using a set of 92 equations popular in the modeling of real world phenomena, as well as linear and polynomial regressions to degree up to 3 . We hand-picked a subset of these models by inspecting plots of their prediction relative to the data (the closer the better), based on their complexity (the least parameters the better), their residual standard error (the least the better), and their $\mathrm{AIC}^{4}$ (smaller the better). We then refined our model selection with a trial-and-error approach consisting of modifying the parameters' start value, iteratively removing parameters

\footnotetext{
${ }^{4}$ Akaike Information Criterion (AIC) [2]
}

with low significance t-tests values, and comparing AIC and residual standard error.

Manual Approach Use. For Manual, we selected a reciprocal quadratic-YD model:

$$
r_{m}=\frac{\mu_{m}}{\theta_{1}+\theta_{2} \cdot \mu_{m}+\theta_{3} \cdot \mu_{m}^{2}}
$$

with parameters $\theta_{1}=0.0630, \theta_{2}=1.01$ and $\theta_{3}=0.0770$. A plot of the residuals revealed a biaised homoscedastic distribution, whose linear pattern may be due to a lurking variable. This lurking variable is unlikely to be ACCURACY as other models that do include it revealed a similar trend. The residual standard error is 0.236 on 774 degrees of freedom, all parameters are significant $\left(p<.01\right.$ for $\theta_{1}$ and $p<.001$ for the two others). The model depends on $\mu_{m}$ only (it still indirectly depends on ACCURACY through $\mu_{m}$ ).

The model is mainly characterized by a descent of the Manual Approach Ratio $r_{m}$ when $\mu_{m}>1$ with a horizontal asymptote at $r_{m}=0$ (see Fig 3): as the number of steps to be taken increases, participants used less and less a manual approach ratio, but it is always used. As $\mu_{m}$ decreases below $0.904, r_{m}$ plunges toward 0 , modeling an abrupt inversion of the manual approach use. This was observed for $s=32$, and both $a=0.7$ and $a=0.9$.

Auto Approach Use. We use the following simplified full quadratic model:

$$
r_{a}=\omega_{1} \cdot \mu_{m}+\omega_{2} \cdot a^{2}+p 3 \cdot \mu_{m} \cdot a+\omega_{4} \cdot \mu_{m} \cdot a^{2}
$$

with parameters $\omega_{1}=-0.00306, \omega_{2}=0.132, \omega_{3}=-0.0629$ and $\omega_{4}=-0.0333$. As with Manual's regression, a plot of the residuals revealed a biaised homoscedastic distribution. The residual standard error is 0.235 on 774 degrees of freedom, all parameters are significant $\left(p<.01\right.$ for $\omega_{4}$, and $p<.001$ for the others).

Mostly, the Auto Approach Ratio $r_{a}$ quadratically increases with $a$, meaning participants increasingly use auto as the automation gets more accurate. However, the slope is linearily tempered by $\mu_{m}$ (see Fig 3): participants use an auto 
Table 1: Statistical significance of the questionnaires

\begin{tabular}{lccccc}
\hline & \multicolumn{2}{c}{ ACCURACY } & & \multicolumn{2}{c}{ STEP } \\
\cline { 2 - 3 } \cline { 5 - 6 } & $\chi^{2}(4)$ & $p$-value & & $\chi^{2}(4)$ & $p$-value \\
\hline NASA-TLX & & & & & \\
$\quad$ Effort & 34.785 & $<.001$ & & 4.799 & .308 \\
Frustration Level & 70.555 & $<.001$ & & 15.568 & $<.01$ \\
Overall Performance & 9.055 & .060 & & 6.495 & .170 \\
Mental Demand & 16.014 & $<.01$ & & 1.861 & .761 \\
Physical Demand & 64.440 & $<.001$ & 29.194 & $<.001$ \\
Temporal Demand & 17.469 & $<.01$ & & 3.911 & .881 \\
Questionnaire & & & & .137 \\
Perceived Accuracy & 211.19 & $<.001$ & & 6.975 & \\
Perceived Controllability & 67.343 & $<.001$ & & 166.63 & $<.001$ \\
Feeling of Control & 23.206 & $<.001$ & 82.351 & $<.001$ \\
Feel. of Accomplishment & 3.249 & .517 & & 11.628 & $<.05$ \\
Feeling of Responsibility & 9.019 & .060 & & 8.997 & .061 \\
Satisfaction & 105.84 & $<.001$ & & 40.197 & $<.001$ \\
Enjoyment & 36.926 & $<.001$ & 13.539 & $<.01^{*}$ \\
\hline
\end{tabular}

*significant Kruskal-Wallis $p$-value, but no pairwise differences detected

rectification approach as the manual approach can be performed in less steps. $\mu_{m}$ 's negative impacts on $r_{a}$ interacts both linearily and quadratically with $a$. This interaction can be explained by the dependence of $\mu_{m}$ on $a$.

Mixed Approach Use. The Mixed Approach Ratio is simply those approaches that were not entirely manual or auto:

$$
r_{x}=1-r_{a}-r_{m}
$$

Overall, Manual Approach topped Auto Approach all along our experiment. It was only overtaken in 4 out of 5 of the lowest STEP conditions. Their intersection can be calculated using our models and do not occur before controllability is very low. Notably $r_{a}=r_{m}$ for $\left\{a=0.1, \mu_{m}=60.5\right\}$ $(s=1.68),\left\{a=0.5, \mu_{m}=18.7\right\}(s=1.01)$ and $\{a=$ $\left.0.9, \mu_{m}=13\right\}(s=0.556)$.

\section{NASA-TLX}

The results of the NASA-TLX scale and our subjective questionnaire are shown in Fig. 4. The results of Kruskal-Wallis significance tests are provided in Table 1 . When an effect was detected, we ran pairwise comparisons using a Wilconxon rank sum test with Bonferroni correction.

Except for Performance, we found an effect of ACcuracy on all NASA-TLX measures. We found significant differences of ACCURACY for each pair under $a=0.5$ (all $p<.05$ ), on all NASA-TLX measures except for Temporal Demand and Performance. On all measures except Performance and Mental Demand, we found significant differences for $a=0.1$ compared to 0.7 or 0.9 (all $p<.05$ ). No pairwise significant differences were detected between $a=0.7$ and 0.9 in any NASA-TLX measures.
Few significant differences were detected between the STEP conditions. Frustration was significantly lower for $s=$ 32 compared to $s=1.6$ or 0.8 (both $p<.05$ ), and Physical Demand was significantly higher for $s=32$ compared to $s=1.6$ or 0.8 (both $p<0.01)$.

\section{Subjective Questionnaire}

We found significant differences for all pairs of ACCURACY conditions in terms of Perceived Acceptability (all $p<.001$ ) except $a=[0.5,0.7]$. For Perceived Controllability, we only found significant differences between all non-adjacent pairs ${ }^{4}$ $(p<.001)$ except $a=[0.3,0.5]$ and $[0.5,0.9]$. In terms of Feeling of Control, we found significant differences between $a=0.1$ and all other conditions (all $p<.01$ ) except $a=0.5$. For Satisfaction, we found significant differences for all nonadjacent pairs ${ }^{4}$ (all $p<.01$ ). Finally, with Enjoyment, we found significant differences between $a=0.1$ and all other conditions (all $p<.01$ ) except $a=0.5$.

We found significant differences for all pairs of STEP conditions for Perceived Controllability (all $p<.001$ ), except for $s=[32,6.4]$ and $s=[6.4,3.2]$. In terms of Feeling of Control, we found significant differences for $s=1.6$ compared all other conditions (all $p<.01$ ) except $s=3.2$, as well as between $s=0.8$ and all other conditions (all $p<.01$ ). For Feeling of Accomplishment, we only found a difference between $s=6.4$ and $0.8(p<.05)$, and for Satisfaction, we found a difference between all non-adjacent pairs ${ }^{4}$ (all $p<.05)$ except $s=[3.2,0.8]$.

\section{DISCUSSION}

Rectification Approaches. We were surprised by the strong resistance of participants to use an auto rectification approach, even under very low controllability conditions. They preferred a manual approach in 21 out 25 of the conditions, regardless of a much lower time efficiency compared to an auto approach. Auto approaches were only more frequent in extreme, low controllability, conditions. In fact, the use of the manual approach was best modeled as a function of the average number of steps it requires, and only indirectly relates to ACCURACY.

Extrapolating on the horizontal asymptote on the right hand side of Manual Approach, one may postulate that there is no natural controllability threshold beyond which manual interaction is so poor that participants entirely stop using it. While any discussion drawn from extrapolation should be taken with caution, this suggests people may always use some manual control when they can. This result tends to confirm one of Shneiderman's main claims [17] and to corroborate Oh et al.'s results [14]: users would rather do the task themselves. This may be a strong argument in favour of

\footnotetext{
${ }^{4}$ For example ACCURACY $a=0.1$ and $a=0.5$ but not $a=0.1$ and $a=0.3$.
} 

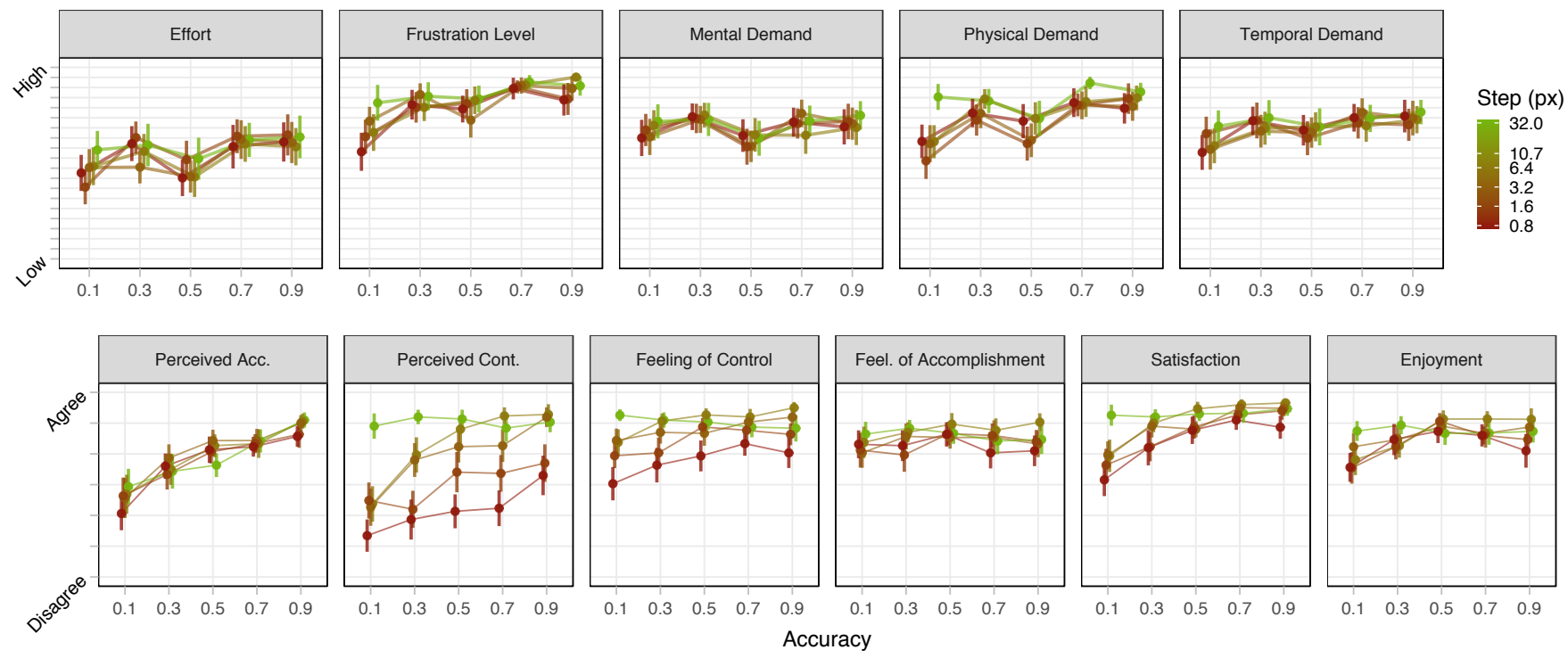

Figure 4: Significant responses of our NASA-TLX and subjective questionnaires.

focusing on controllability before accuracy during the design of automated tasks.

Surprisingly, the equation modelling a manual approach shows an abrupt drop of its use when the expected number of steps to be taken passes below $\mu=0.90$. An explanation to this phenomenon comes from the fact that at this point, usage of the manual rectification interaction is so uncommon that users may forget its existence. Paradoxically, under very high accuracy and controllability conditions, a slightly lower controllability makes the need for it even more obvious to participants, and consequently it actually increases its use.

Subjective Perception of the Task. Except performance, all NASA-TLX measures significantly increase with higher accuracy (see Table 1 and Fig. 4). This is a strong argument for focusing on accuracy: low accuracy values are effectively perceived as less usable. However, the slopes tend to plateau after $a=0.7$, hinting that after reaching this threshold, improving accuracy made little difference in terms of usability. On the other hand, few significant differences could be detected for the controllability factor STEP.

We observed surprising significant lower values in all NASA-TLX measures for $a=0.5$. Its reason remains uncertain. Our hypothesis is that $50 \%$ accuracy is an uncomfortably balanced spot where users cannot anticipate the outcome of the automation: correct and incorrect are equiprobable.

Interestingly, the behaviour of the step $s=32$ curve seems to follow a slightly different pattern compared to the others. For example, while Perceived Controllability always increases with ACCURACY, in the other STEP conditions, it decreases instead with $s=32$. This may be related to the drop in Manual Approach discussed above, and may be explained with the same reason. It also exposes a quite interesting trend on the Satisfaction measure. For $s=32$, participant's satisfaction is essentially constant, while it descends with ACCURACY on the other conditions.

Satisfaction virtually always increases with STEP, even for high ACCURACY: except for all adjacent STEP values (like $s=6.4$ and 3.2) always increases. This seems to indicate that increasing controllability is virtually always worth it. A similar reasoning led to the same conclusion with ACCURACY, indicating that both matter to a user's satisfaction. Even with low accuracy, self-reported satisfaction remains constant for high controllability conditions, and vice versa.

Limitations. For usage measures, we formally modeled the relationship between controllability and accuracy. This is ambitious and difficult. As with most real phenomena, entirely capturing its complexity is outside a model's reach, and its understanding can only be acquired iteratively. As a result, some results necessarily include caveats.

Specifically, it focuses on one particular form of relationship stripped down to its bare minimum: an instance where the result of an automation run is independent from the previous one, and where the amount of inaccuracies of the automation-and consequently the amount of effort to rectify it-is normally distributed. It does not capture factors like managing subtasks, or consequences of task performance. Also, we manipulated only two aspects of controllability, namely effort and time with all others held constant. Finally, while we mainly discuss our results in term of STEP and effort as a factor of controllability, STEP linearly impacts time, which is another of factor of controllability. Consequently, our results may be caused by effort, time, or both. 


\section{CONCLUSION}

This work is the first to formally investigate the relationship between automation accuracy and controllability of an automated task. We formalized the concept of controllability, and our controlled experiment showed how controllability impacts user satisfaction, and how it is (or is not) affected by automation accuracy. Our results also revealed a strong preference for manual rectification after automation, even under very poor controllability and high accuracy.

These results suggest future work to investigate more aspects of this space. For example, exploring how cognitive load, or time independent from effort, co-mingle with controllability and accuracy. It would also be valuable to investigate how the controllability-accuracy relationship impacts trust, or how people adapt their behaviour after longer exposure to a system with a certain level of controllability and accuracy.

With a more nuanced examination of human behaviour in a controlled task, we have a better understanding of the automation-controllability trade-off. This has direct implications for the design of automated tasks, and it could justify prioritising decisions and resources for industry and research. We also hope this nudges automation researchers to the idea that users not only can, but want to contribute to the success of an automated task. A well designed user-in-the-loop approach is a valid automation solution that will likely result in higher user satisfaction.

\section{ACKNOWLEDGEMENTS}

This work was supported by Huawei Noah’s Ark Lab.

\section{REFERENCES}

[1] Ashraf Abdul, Jo Vermeulen, Danding Wang, Brian Y Lim, and Mohan Kankanhalli. 2018. Trends and Trajectories for Explainable, Accountable and Intelligible Systems: An HCI Research Agenda. In Proceedings of the $2018 \mathrm{CHI}$ Conference on Human Factors in Computing Systems (CHI '18). ACM, New York, NY, USA, 582:1-582:18. https://doi.org/10.1145/3173574.3174156

[2] Hiroyuki Akaike. 1974. A new look at the statistical model identification. IEEE Trans. Automat. Control 19, 6 (dec 1974), 716-723. https://doi.org/10.1109/TAC.1974.1100705

[3] Michel Beaudouin-Lafon. 2004. Designing interaction, not interfaces. In Proceedings of the Working Conference on Advanced Visual Interfaces (AVI '04). ACM, New York, NY, USA, 15-22. https://doi.org/10.1145/ 989863.989865

[4] John P. Chin, Virginia A. Diehl, and Kent L. Norman. 1988. Development of an Instrument Measuring User Satisfaction of the Humancomputer Interface. In Proceedings of the SIGCHI Conference on Human Factors in Computing Systems (CHI '88). ACM, New York, NY, USA, 213-218. https://doi.org/10.1145/57167.57203

[5] Fred D. Davis. 1989. Perceived Usefulness, Perceived Ease of Use, and User Acceptance of Information Technology. MIS Q. 13, 3 (sep 1989), 319-340. https://doi.org/10.2307/249008

[6] John D. Gould and Clayton Lewis. 1985. Designing for usability: key principles and what designers think. Commun. ACM 28, 3 (1985), 300-311.
[7] Sandra G. Hart and Lowell E. Staveland. 1988. Development of NASATLX (Task Load Index); Results of Empirical and Theoretical Research. In Human Mental Workload, Peter A Hancock and Najmedin Meshkati (Eds.). Advances in Psychology, Vol. 52. North-Holland, 139-183. https: //doi.org/10.1016/S0166-4115(08)62386-9

[8] Keiko Katsuragawa, Ankit Kamal, and Edward Lank. 2017. Effect of Motion-Gesture Recognizer Error Pattern on User Workload and Behavior. Proceedings of the 22nd International Conference on Intelligent User Interfaces - IUI '17 (2017), 439-449. https://doi.org/10.1145/ 3025171.3025234

[9] Matthew Kay, Shwetak N. Patel, and Julie A. Kientz. 2015. How Good is 85\%?: A Survey Tool to Connect Classifier Evaluation to Acceptability of Accuracy. Proceedings of the ACM Conference on Human Factors in Computing Systems (CHI 2015) (2015), 347-356. https://doi.org/bqd5

[10] Jaron Lanier. 1995. Agents of Alienation. interactions 2, 3 (jul 1995), 66-72. https://doi.org/10.1145/208666.208684

[11] Kenneth Levenberg. 1944. A Method for the Solution of Certain NonLinear Problems in Least Squares. Quart. Appl. Math. 2, 2 (1944), 164-168. http://www.jstor.org/stable/43633451

[12] Pattie Maes. 1994. Agents That Reduce Work and Information Overload. Commun. ACM 37, 7 (jul 1994), 30-40. https://doi.org/10.1145/ 176789.176792

[13] Donald Marquardt. 1963. An Algorithm for Least-Squares Estimation of Nonlinear Parameters. F. Soc. Indust. Appl. Math. 11, 2 (1963), 431441. https://doi.org/10.1137/0111030

[14] Changhoon Oh, Jungwoo Song, Jinhan Choi, Seonghyeon Kim, Sungwoo Lee, and Bongwon Suh. 2018. I Lead, You Help but Only with Enough Details: Understanding User Experience of Co-Creation with Artificial Intelligence. In Proceedings of the 2018 CHI Conference on Human Factors in Computing Systems (CHI '18). ACM, New York, NY, USA, 649:1-649:13. https://doi.org/10.1145/3173574.3174223

[15] Chris Olah, Arvind Satyanarayan, Ian Johnson, Shan Carter, Ludwig Schubert, Katherine Ye, and Alexander Mordvintsev. 2018. The Building Blocks of Interpretability. Distill (2018). https://doi.org/10.23915/ distill.00010

[16] Danny Plass-Oude Bos, Bram van de Laar, Boris Reuderink, Mannes Poel, and Anton Nijholt. 2014. Perception and Manipulation of Game Control. Lecture Notes of the Institute for Computer Sciences, SocialInformatics and Telecommunications Engineering, LNICST 136 LNICST (2014), 57-66. https://doi.org/10.1007/978-3-319-08189-2 7

[17] Ben Shneiderman. 1993. Beyond Intelligent Machines: Just Do It! IEEE software 10, 1 (1993), 100-103.

[18] Ben Shneiderman. 1995. Looking for the Bright Side of User Interface Agents. interactions 2, 1 (jan 1995), 13-15. https://doi.org/10.1145/ 208143.208150

[19] Ben Shneiderman. 1997. Designing the User Interface: Strategies for Effective Human-Computer Interaction (3rd ed.). Addison-Wesley Longman Publishing Co., Inc., Boston, MA, USA.

[20] Ben Shneiderman and Pattie Maes. 1997. Direct Manipulation vs. Interface Agents. Interactions 4, 6 (nov 1997), 42-61. https://doi.org/ $10.1145 / 267505.267514$

[21] Hyewon Suh, Nina Shahriaree, Eric B. Hekler, and Julie A. Kientz. 2016. Developing and Validating the User Burden Scale: A Tool for Assessing User Burden in Computing Systems. In Proceedings of the 2016 CHI Conference on Human Factors in Computing Systems (CHI '16). ACM, New York, NY, USA, 3988-3999. https://doi.org/10.1145/ 2858036.2858448

[22] Zheng Lu, Zheng Wu, and Michael S. Brown. 2009. Interactive degraded document binarization: An example (and case) for interactive computer vision. In 2009 Workshop on Applications of Computer Vision (WACV). IEEE, 1-8. https://doi.org/10.1109/WACV.2009.5403091 